Article

\title{
Conformation of the Intermediates in the Reaction Catalyzed by Protoporphyrinogen Oxidase: An In Silico Analysis
}

\author{
Abigail L. Barker (D), Hamlin Barnes and Franck E. Dayan *(i) \\ Agricultural Biology Department, Colorado State University, Fort Collins, CO 80523, USA; \\ abigail.barker@colostate.edu (A.L.B.); ruth.enocene@yahoo.com (H.B.) \\ * Correspondence: franck.dayan@colostate.edu; Tel.: +1-662-816-6214
}

Received: 6 November 2020; Accepted: 10 December 2020; Published: 14 December 2020

\begin{abstract}
Protoporphyrinogen oxidase (PPO) is a critical enzyme across life as the last common step in the synthesis of many metalloporphyrins. The reaction mechanism of PPO was assessed in silico and the unstructured loop near the binding pocket was investigated. The substrate, intermediates, and product were docked in the catalytic domain of PPO using a modified Autodock method, introducing flexibility in the macrocycles. Sixteen PPO protein sequences across phyla were aligned and analyzed with Phyre2 and ProteinPredict to study the unstructured loop from residue 204-210 in the $H$. sapiens structure. Docking of the substrate, intermediates, and product all resulted in negative binding energies, though the substrate had a lower energy than the others by $40 \%$. The $\alpha-\mathrm{H}$ of $\mathrm{C} 10$ was found to be 1.4 angstroms closer to FAD than the $\beta-\mathrm{H}$, explaining previous reports of the reaction occurring on the meso face of the substrate. A lack of homology in sequence or length in the unstructured loop indicates a lack of function for the protein reaction. This docking study supports a reaction mechanism proposed previously whereby all hydride abstractions occur on the C10 of the tetrapyrrole followed by tautomeric rearrangement to prepare the intermediate for the next reaction.
\end{abstract}

Keywords: reaction mechanism; docking of tetrapyrrole macrocycles; Lennard-Jones forces

\section{Introduction}

The porphyrin pathway plays a central role in the synthesis of many pigments fundamental to sustaining life across the biological realm [1-3]. Macrocyclic tetrapyrroles derived for this pathway have a varying degree of conjugation and can be chelated with metal dications such as the alkaline earth metal $\mathrm{Mg}^{2+}$, transition metals such as $\mathrm{Fe}^{2+}, \mathrm{Co}^{2+}$, and $\mathrm{Ni}^{2+}$, and group 12 element $\mathrm{Zn}^{2+}$. These metals are coordinated with the pyrrole nitrogens at the center of these rings. Examples of biologically important metalloporphyrins include chlorophylls $\left(\mathrm{Mg}^{2+}\right)$, hemes $\left(\mathrm{Fe}^{2+}\right)$, vitamin $\mathrm{B} 12\left(\mathrm{Co}^{2+}\right)$, and coenzyme F430 $\left(\mathrm{Ni}^{2+}\right)$.

Biosynthesis of porphyrins consists of 7 core enzymes that culminates in the action catalyzed by protoporphyrinogen oxidase (PPO) converting the colorless precursor protoporphyrinogen IX (protogen) into the fully conjugated, bright red pigment protoporphyrin IX (proto) [3]. PPO itself is of particular interest because mutations of the protein lead to variegate porphyria diseases in humans and in agriculture it is the protein target of the PPO-inhibiting herbicides which are seeing a resurgence of use in recent years [4,5].

There have been many inquiries into the mechanism of the three sequential oxidation reactions which convert protogen into the fully conjugated proto. An early study of the biosynthesis of heme using isotopically labelled tetrapyrrole determined that the reaction involved hydride abstractions from three of bridging methylene groups [6]. This mechanism posited two significant mechanistic 
properties: the hydride ion was abstracted from the meso face $(\alpha-\mathrm{H})$ and tautomerism around the neighboring tetrapyrrole ring resulted in the formation of a double bond (Figure 1).

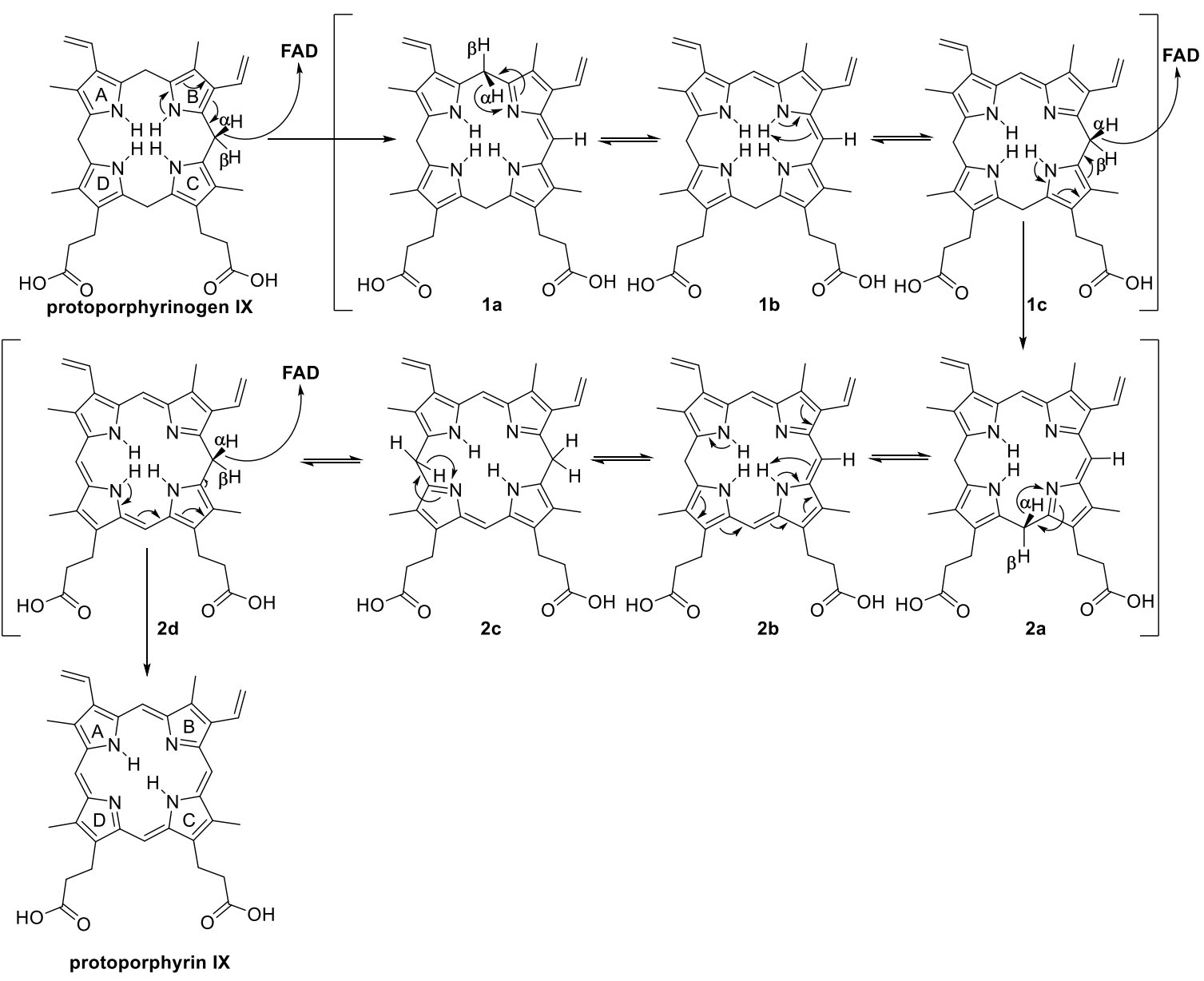

Figure 1. Putative reaction intermediates involved in the catalytic reaction of PPO converting protogen and proto. This figure is adapted from reaction scheme proposed by Koch et al. [7] but orienting the tetrapyrrole ring to match that of copro in its binding site in uroporphyrinogen decarboxylase (1R3Y) [8].

This mechanism required that protogen and the intermediates rotate within the catalytic domain so that the meso face of each methylene functionality was exposed to FAD for the next hydride abstraction to occur. However, elucidation of PPO's structure by X-ray crystallography suggested that protogen is held in a relatively fixed position within the catalytic domain by having the propionate group from ring $\mathrm{D}$ (Figure 1) interacting with the guanidino functional group of Arg97 and maintaining the $\mathrm{C} 10$ carbon of protogen in close proximity to N5 of the cofactor FAD [7]. Still, no published crystal structure of PPO has been reported with either the substrate or the product in the active site. A previous effort to bind protogen to PPO in silico was performed with the structure of protogen created by an extensive analysis process and a structure of PPO which was allowed to relax in a molecular dynamics simulation [9]. This method does not take into account the structure of the precursors in the pathway which are unlikely to change due to the repulsion of the hydrogen molecules in the center of the tetrapyrrole ring, and new methods with the Autodock program have simplified the analysis of flexible substrates such as protogen.

The most current understanding of PPO's reaction mechanism involves 3 subsequent hydride abstractions on $\mathrm{C} 10$ of protogen, the methylene bridge between pyrrole rings $\mathrm{B}$ and $\mathrm{C}$, followed by tautomerization to regenerate the unsaturated state of C10 allowing for the next reaction [7]. Mechanistically, the first reaction is initiated by a 1:1 stoichiometric FAD as a coenzyme. The methylene functionality is prochiral because the $\alpha-\mathrm{H}$ is much closer to N5 of FAD than the $\beta-\mathrm{H}$ (Figure 1). 
Once the meso hydrogen is removed in the form of a hydride ion, it creates an unstable carbocation transition state at C10, which instantly rearranges in ring B as shown in intermediate 1a in Figure 1. The second reaction is an enamine-imine tautomerization from $1 \mathrm{~b}$ to $1 \mathrm{c}$ resulting in the reformation of the methylene bridge at $\mathrm{C} 10$ by transfer of the proton from ring B pyrrole nitrogen. $\mathrm{FADH}^{-}$is oxidized to FAD by reaction with $\mathrm{O}_{2}$ and the proton released from pyrrole $\mathrm{N}$ in the third reaction and generates $\mathrm{H}_{2} \mathrm{O}_{2}$. The second step repeats the same sequence of reactions. Oxidation of $\mathrm{C} 10$ by FAD, $1 \mathrm{c}$ and instant rearrangement of ring $C, 2 a$ followed by the enamine-imine tautomerization $2 b$ to $2 c$ and the subsequent reoxidation of $\mathrm{FADH}^{-}$to FAD. The third step involves the oxidation of $\mathrm{C} 10$ by FAD and four two electron rearrangements from ring $\mathrm{D}, 2 \mathrm{~d}$ to yield proto.

Multiple crystal structures of PPO from differing organisms show similar folding despite a large variation in conservation of sequence: from around $90 \%$ identity between mammals to as low as $23 \%$ identity between mammalian and plant sequences (Supplementary Table S1). There are two separate isoforms of PPO in plant species which only share about $27 \%$ sequence identity, generally considered the chloroplastic and mitochondrial isoforms. Along with the mostly conserved structure, some conserved residues across phyla include the Arg97 region and the Phe353-Leu356 region [4]. One conserved feature is an unstructured loop that is not captured in any of the published crystal structures that is adjacent to the catalytic domain of the protein, located from residue 204 to 210 in the human PPO. The flexible nature of the loop and proximity to the reaction pocket could signify importance if these sequences are conserved across phyla.

We aim to better understand the reaction catalyzed by PPO by docking the substrate and product of the reaction along with relevant reaction intermediates, describe the reaction mechanism in a way that unifies previous data while taking into account the spatial limitation of the catalytic domain of PPO as well as information from substrate orientations upstream in the pathway, and to determine if sequence similarity suggests that the unstructured loop has a function in the reaction or substrate binding.

\section{Results and Discussion}

\subsection{Determining the Initial Conformation of Protogen}

Protogen is a very flexible tetrapyrrole because each pyrrole ring is connected to the other via unsaturated methylene bridges. There is no published crystal structure of protogen cocrystallized with any protein, and consequently it is difficult to predict the conformation of protogen in PPO. Free protogen creates a dramatically bent shape naturally because of the repelling forces between the center hydrogen atoms. This natural shape does not fit in the catalytic domain of PPO. Furthermore, there is evidence of protein channeling or chaperoning of the intermediates in this pathway $[7,10,11]$. Therefore, we obtained a realistic starting conformation from the structure of coproporphyrinogen III (copro), which contains the same repelling hydrogens and flexible methylene bridges, cocrystallized with uroporphyrinogen decarboxylase (1R3Y) [8]. Since the only difference between copro and protogen is the presence of propionic acids instead of vinyl groups on rings A and B (Figure 2), protogen was constructed from the coordinate of the optimized structure of copro. This slightly concave conformation is a particularly good starting point for docking protogen and the reaction intermediates because it fits relatively well in the catalytic domain of PPO.

The structures of all the subsequent reaction intermediates were derived from this original confirmation by editing the mol 2 files followed by geometric optimization with Spartan 18 according to the putative reaction scheme proposed by Koch et al. [7] (Figure 1). 

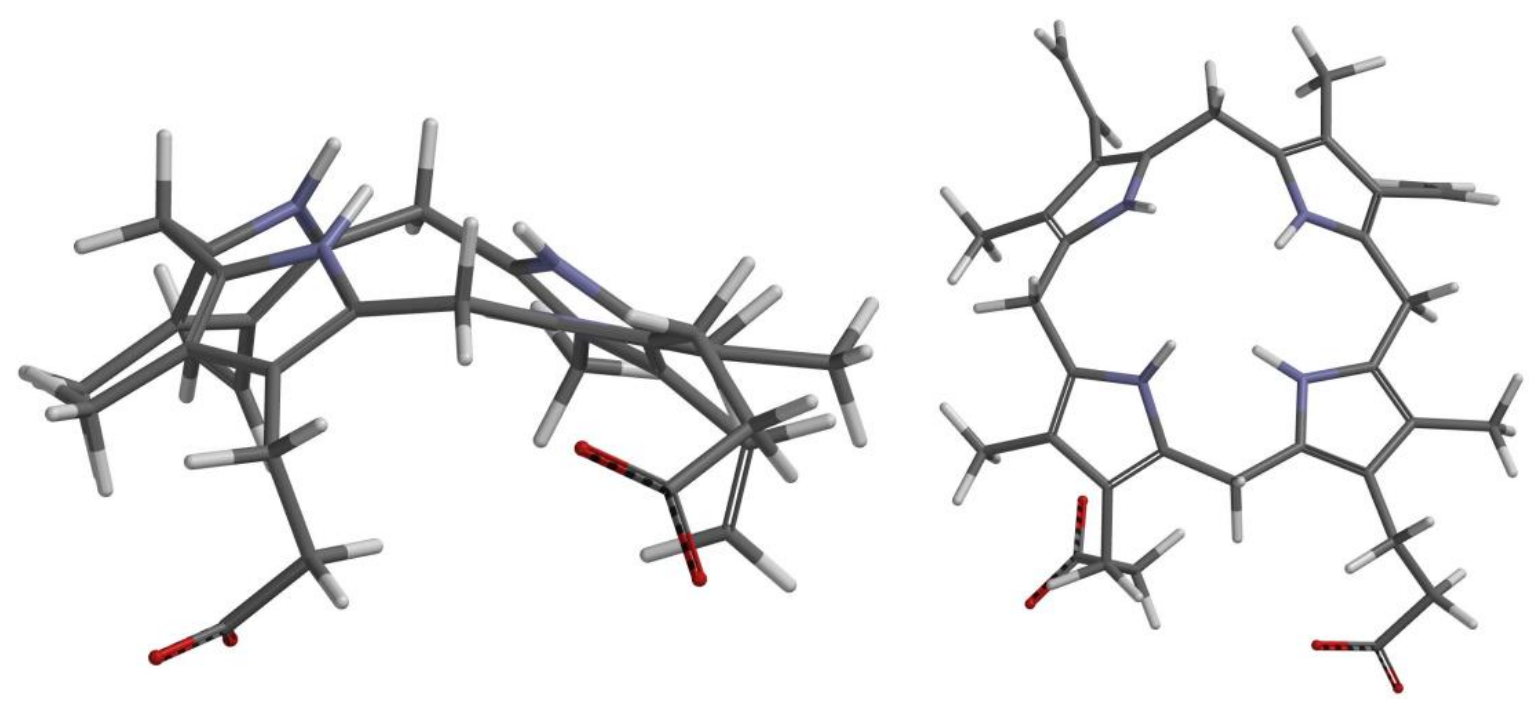

Figure 2. Starting conformation of geometrically optimized protogen based on the coordinates of copro in uroporphyrinogen decarboxylase (1R3Y) [8].

\subsection{Docking of Protogen, Tautomeric Reaction Intermediates and Proto to PPO}

The reaction catalyzed by PPO involves 3 oxidative steps on the same carbon (C10) forming the methylene bridge between the $\mathrm{B}$ and $\mathrm{C}$ pyrrole rings followed by tautomeric rearrangements (Figure 1 ). In order to dock protogen and all the relevant reaction intermediates in PPO, the docking protocol for Autodock was modified to allow flexibility of the tetrapyrrole macrocycles according to the method developed by Forli [12,13]. The method consists of 'breaking' one of the bonds in the tetrapyrrole rings, replacing carbons at dummy atoms and applying a Lennard-Jones force between these dummy atoms to keep them proximal to each other during the docking procedure. Accordingly, we avoided bonds in partially rigid regions to reduce the complexity and calculation time without compromising accuracy. Consequently, the bond between the methylene carbon between rings $C$ and D was selected (Figure 3) since it is the most flexible bond throughout all the steps shown in Figure 1, due to the fact that it is the last single bond to be oxidized. This method creates a flexible ring system with an increased number of rotatable bonds to be considered during calculations.

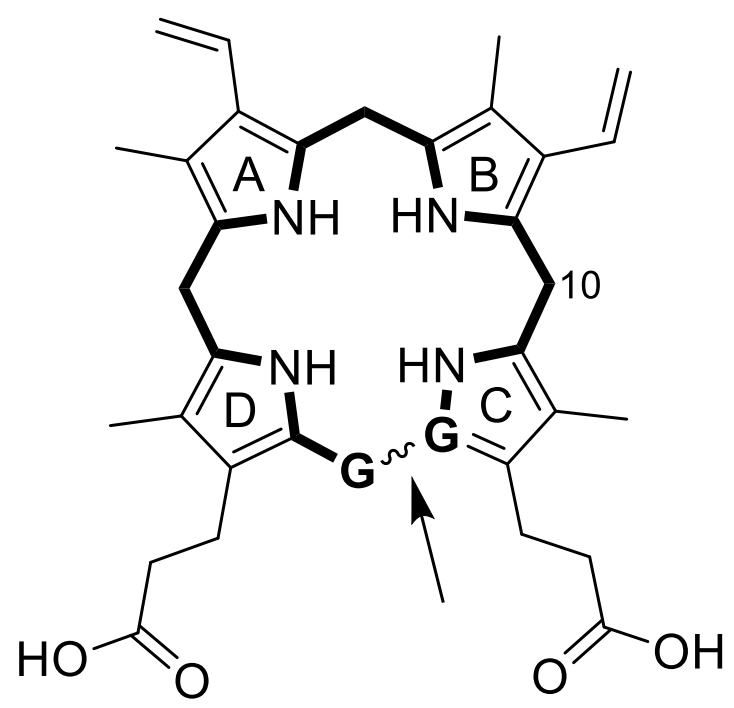

Figure 3. Localization of the broken bond (arrow) and respective dummy atoms (labeled G) selected to allow docking of the flexible ring system of the tetrapyrrole intermediates in the conversion of protogen to the last tautomer before proto. The carbon (C10) where the hydride transfers occur is labelled. 
Proto was obtained from x-ray analysis of protoporphyrin IX dimethyl ester by removing the methyl esters. The fully conjugated product of the reaction catalyzed by PPO is known to be a non-flexible planar structure and was docked in PPO without breaking the cyclic tetrapyrrole. The location of the catalytic domain was defined using a gridbox that encompassed the FAD forming the 'roof' of the cavity and, the top of $\alpha-8$ helix of PPO upon which the tetrapyrrole rings are centered at the 'bottom' of the cavity, and Arg97 involved in stabilizing the rings on the right side of the cavity (Supplemental Figure S1).

After the docking, RMSD of the clusters with best poses for each tetrapyrrole intermediate provide insight to the changes in the curvature of the tetrapyrroles during the oxidation between protogen and proto (Figure 4). The RMSD for protogen is small, relative to the other reaction intermediates 1a, 1c, 2a and $2 \mathrm{~d}$. The low RMSD of protogen is most likely a reflection of the flexibility afforded by the fully unsaturated methylene bridges linking the pyrrole rings. In fact, the movement of the propionate side chains probably accounts for a significant portion of the RMSD. On the other hand, the increased RMSD of the reaction intermediates is due to the increased rigidity and planarity of the macromolecules as additional double bonds are introduced in the methylene bridges. The RMSD of the proto is also low. It should be noted that this refers to the coordinates of the crystal structure published by Caughey and Ibers [14]. Since proto retained its planar conformation relative to the starting conformer used for docking, little change in the shape of the tetrapyrrole ring is expected. As with protogen, a significant portion of the RMSD is probably due the movement of the propionate side chains.

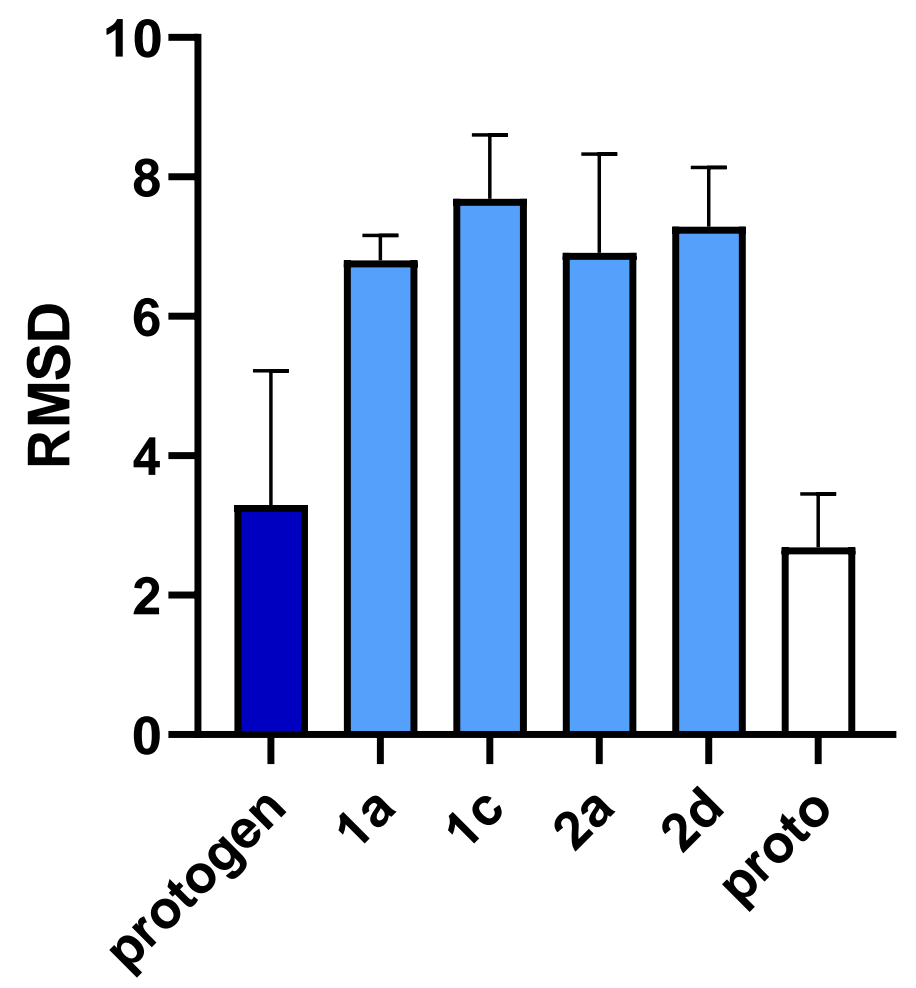

Figure 4. RMSD of the clusters with best poses for each tetrapyrroles relative to the conformations of the starting conformers prior to docking. Note that the starting conformers for protogen, 1a, 1c, 2a and $2 \mathrm{~d}$ were designed using the coordinates of coproporphyrinogen bound within uroporphyrinogen carboxylase (1R3Y) [8], whereas the starting conformer of proto is based on the published planar coordinate of this molecule [14].

When docked in the catalytic domain the binding energy of protogen averaged $-10.59 \pm 1.08$ $\mathrm{kcal} / \mathrm{mol}$ (Figure 5). The binding energies of all reaction intermediates and proto were on average $40 \%$ greater than that of protogen, ranging from -6.00 to $-7.27 \mathrm{kcal} / \mathrm{mol}$. This is accounted for by the fact that protogen is the most flexible of the tetrapyrroles, having 4 saturated methylene bridges connecting each 
pyrrole rings. All subsequent tetrapyrroles are less flexible because of the introduction of an increasing number of unsaturated methylene bridges, leading to the fully conjugated and least flexible product of the reaction (proto). These binding energies agree with the paradigm of PPO-protogen $>$ PPO-proto established in Hao et al. [9].

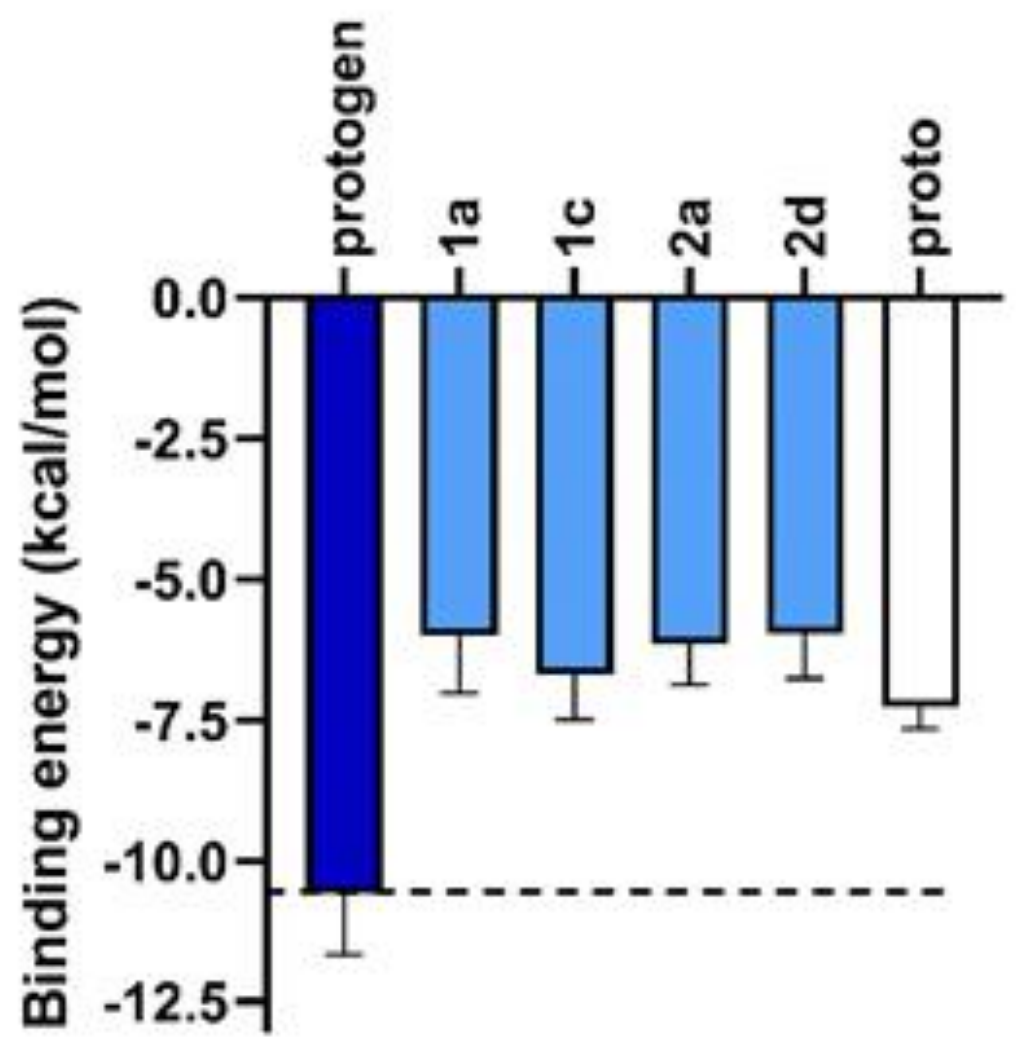

Figure 5. Binding energy ( $\mathrm{kcal} / \mathrm{mol}$ ) of protogen (dark blue bars), reactions intermediates (light blue bars) and proto (white bars) to human PPO. The more negative the binding energy reflects a better fit within the catalytic domain. Dotted line represents the average binding energy of protogen.

The docking protocol allowed for the propionate groups of either ring $\mathrm{C}$ or $\mathrm{D}$ to interact with the guanidino functional group of Arg97 (Figure 6), all the docked molecules from cluster 1 assumed the expected pose with the propionate group from ring D interacting with and maintaining the $\mathrm{C} 10$ carbon of protogen in close proximity to N5 of the cofactor FAD (Figure 6) [7]. On average the propionate group was between 4.3 and $4.6 \AA$ from Arg 97 and the C10 of protogen (and the reaction intermediates) was between 3.3 and $4.1 \AA$ from N5 of FAD (Table 1).

Table 1. Summary of the parameters associated with the molecules docked in the expected orientation in PPO.

\begin{tabular}{|c|c|c|c|}
\hline & $\begin{array}{l}\text { Selected Docked } \\
\text { Molecules }^{1}\end{array}$ & $\begin{array}{c}\text { Distance between N5 of } \\
\text { FAD and C10 of } \\
\text { Tetrapyrrole }(\AA)\end{array}$ & $\begin{array}{c}\text { Distance between } \\
\text { Propionate Group and } \\
\text { Arg97 (̊̊) }\end{array}$ \\
\hline Protogen & 23 & $4.4 \pm 0.5$ & $3.3 \pm 0.8$ \\
\hline $1 \mathrm{a}$ & 22 & $4.3 \pm 0.7$ & $3.5 \pm 0.9$ \\
\hline $1 \mathrm{c}$ & 12 & $4.3 \pm 0.5$ & $3.3 \pm 0.5$ \\
\hline $2 \mathrm{a}$ & 17 & $4.5 \pm 0.6$ & $3.7 \pm 1.3$ \\
\hline $2 d$ & 20 & $4.3 \pm 0.5$ & $4.1 \pm 1.7$ \\
\hline Proto & 26 & $4.6 \pm 0.2$ & $4.1 \pm 0.8$ \\
\hline
\end{tabular}

${ }^{1}$ Total \# of docked molecules: $n=50$. 

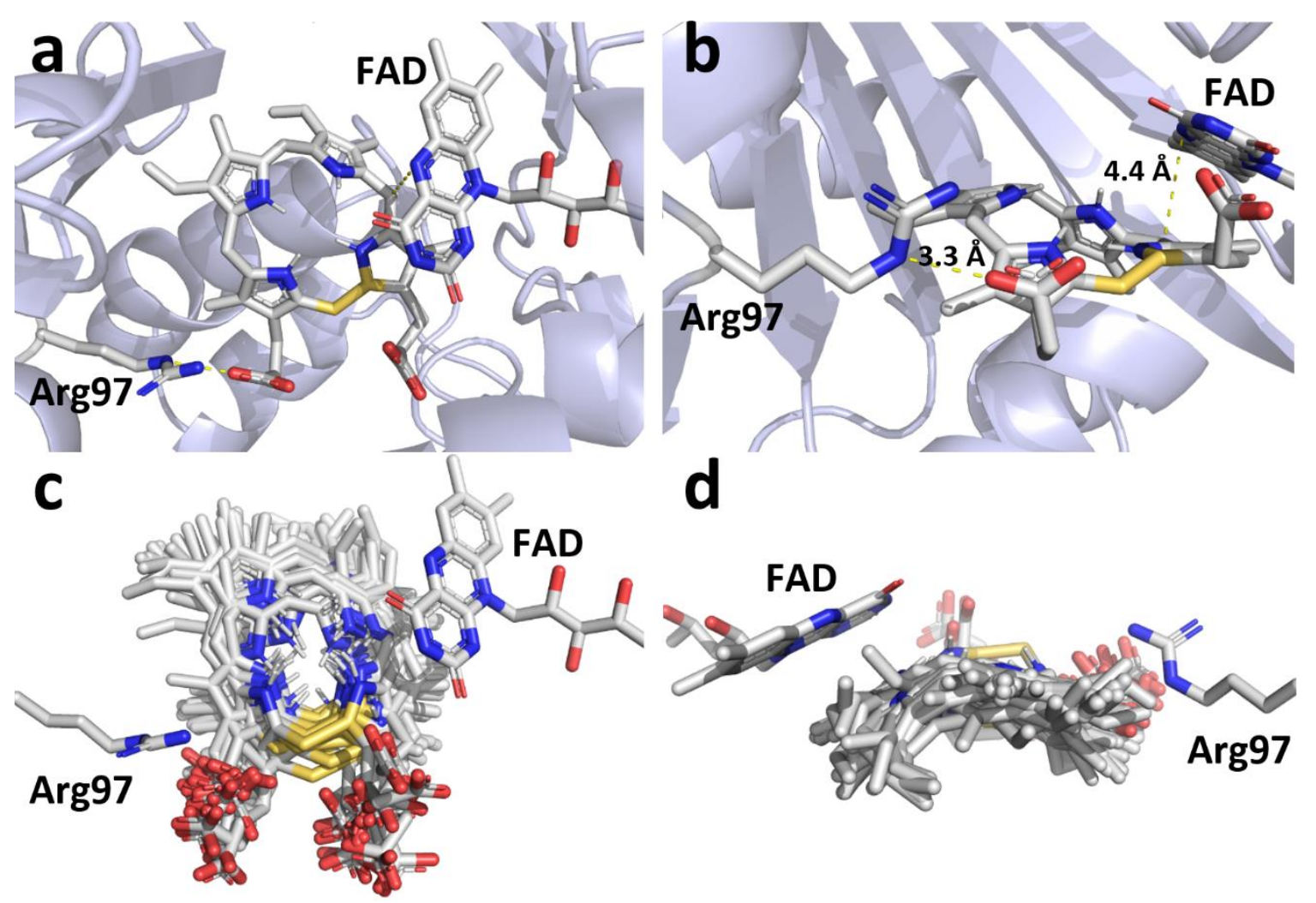

Figure 6. Docking of protogen to PPO using Autodock with Lennard-Jones forces. Similar results were obtained with other reaction intermediates (Supplemental Figures S2-S6). View of the docked protogen with the most favorable binding energy (a) from above and (b) from the opening of the catalytic domain. All of the top docking orientation group are shown to indicate flexibility within the pocket from above (c) and from the back of the pocket (d). Yellow dotted lines in (a) and (b) represent the interactions between protogen and PPO. The average distances between the N5 of FAD and C10 of protogen and between the propionate group of ring D and the Arg97 of PPO are shown in panel (b). Gold bonds represent the location of the Lennard-Jones forces between two dummy atoms.

A key feature of the reaction postulated by Koch et al. [7] is that the oxidation of protogen to proto involves three sequential hydride abstraction from $\mathrm{C} 10$ of the substrate and subsequent reaction intermediate catalyzed at the N5 of FAD. Our docking study supports this by consistently positioning the $\alpha-\mathrm{H}$ from the $\mathrm{C} 10$ of the tetrapyrroles facing the re-face of N5 of FAD. Furthermore, the average distance between N5 and $\alpha-\mathrm{H}$ range from 3.6 to $3.7 \AA$ (Table 2), which is within the known range for redox reactions catalyzed by FAD enzymes [15-21]. On the other hand, the $\beta-\mathrm{H}$ are on average $1.4 \AA$ further from the N5 of FAD (range from 5.0-5.1 $\AA$ ). The second step requires tautomerization with an adjacent pyrrole ring and subsequent loss of a proton from the pyrrole nitrogen, to regenerate the sp3 carbon at $\mathrm{C} 10$ prior to the next hydride abstraction. Experiments using isotopically labeled substrates suggested that the $\alpha$-Hs were preferentially removed during the oxidation of protogen into proto (Figure 1) [6]. Docking of protogen and the reaction intermediates revealed that the poses with the lowest binding energy had the tetrapyrrole rings in a slightly concave conformation for optimal fit within the catalytic domain of PPO (Figure 6). In this conformation, the $\alpha-\mathrm{Hs}$ are closer than the $\beta$-Hs to the adjacent pyrrole bond involved in the tautomerization (Table 2), which accounts for the preferential loss of the meso hydrogen reported by Jones et al. [6]. 
Table 2. Distance between Summary of the parameters associated with the molecules docked in the expected orientation in PPO.

\begin{tabular}{|c|c|c|c|c|}
\hline & \multicolumn{2}{|c|}{$\begin{array}{l}\text { Distance between C10 Methylene H } \\
\text { and N5 of FAD (A) }{ }^{1}\end{array}$} & \multicolumn{2}{|c|}{$\begin{array}{c}\text { Distance between Methylene } \mathrm{H} \text { and } \\
\text { Pyrrole } N(\AA)^{1}\end{array}$} \\
\hline & $\alpha-H$ & $\beta-H$ & $\alpha-H$ & $\beta-H$ \\
\hline Protogen & $3.7 \pm 0.8$ & $5.1 \pm 0.7$ & - & - \\
\hline $1 \mathrm{a}$ & - & - & $3.0 \pm 0.1$ & $3.3 \pm 0.1$ \\
\hline $1 c$ & $3.6 \pm 0.6$ & $5.0 \pm 0.8$ & - & - \\
\hline $2 a$ & - & - & $2.9 \pm 0.2$ & $3.3 \pm 0.1$ \\
\hline $2 d$ & $3.6 \pm 0.6$ & $5.0 \pm 0.7$ & - & - \\
\hline
\end{tabular}

1 Refer to Figure 1 for position of $\alpha-\mathrm{H}$ and $\beta-\mathrm{H}$ on $\mathrm{C} 10$ in protogen and intermediates $1 \mathrm{c}$ and $2 \mathrm{~d}$ involved in the hydride abstraction, and on the methylene carbons on intermediates $1 \mathrm{a}$ and $2 \mathrm{a}$ involved in initiating the tautomerization restoring the $\mathrm{sp} 3$ state of $\mathrm{C} 10$ prior to the next oxidation.

Hydride abstractions are involved in many enzymatic reactions [21-23]. However, the mechanisms involved in hydride transfers had never been demonstrated empirically in biological systems until the recent groundbreaking work on protochlorophyllide oxidoreductase [24]. While that study involves a tetrapyrrole molecule downstream from PPO, the enzyme being considered herein, the reaction is not identical. In the case of protochlorophyllide oxidoreductase, the cofactor is NADPH (instead of FAD), and the direction of the hydride transfer is from the cofactor to ring $\mathrm{D}$ of the substrate, rather than from the methylene bridge between ring B and C of the substrate to the cofactor. Nevertheless, this new understanding of hydride transfer mechanisms provides some relevant insight in the reaction catalyzed by PPO. Indeed, the participation of FAD in a wide range of redox reactions is well documented [23,25]. In particular, the N5 of the oxidized tricyclic isoalloxazine ring system is often a site for hydride addition, converting FAD to $\mathrm{FADH}^{-}$[26].

\subsection{Characterization of the Disordered Loop Crossing the Opening of PPO Binding Domain}

Following the docking, an alignment was created of the top 80 related sequences to the human PPO sequence from the crystal structure used for this research to investigate the unstructured, flexible loop region near the catalytic domain, from residues 204-210. The flexibility and proximity could indicate that the loop has a function in the binding of the substrate or the release of the product. Residues that are conserved in homologous proteins between species and phyla are often associated with important function [27]. Alignment of the top 80 sequences showed a lack of homology from residue 205-209 which covers the entire sequence that is not captured in crystal structures. Furthermore, in the alignment of sequences across phyla the loop is not only not conserved at the residue level but varies in length as well (Figure 7). In protein structure predictions, across all species, the region is considered a solvent exposed region and a disordered region showing no defined secondary structure. Based on these observations, it is unlikely that the region is directly involved with the binding of the substrate or the reaction carried out by PPO. Conservation of the flexible region could indicate the necessity of movement near the catalytic domain to accommodate substrate and product movement.
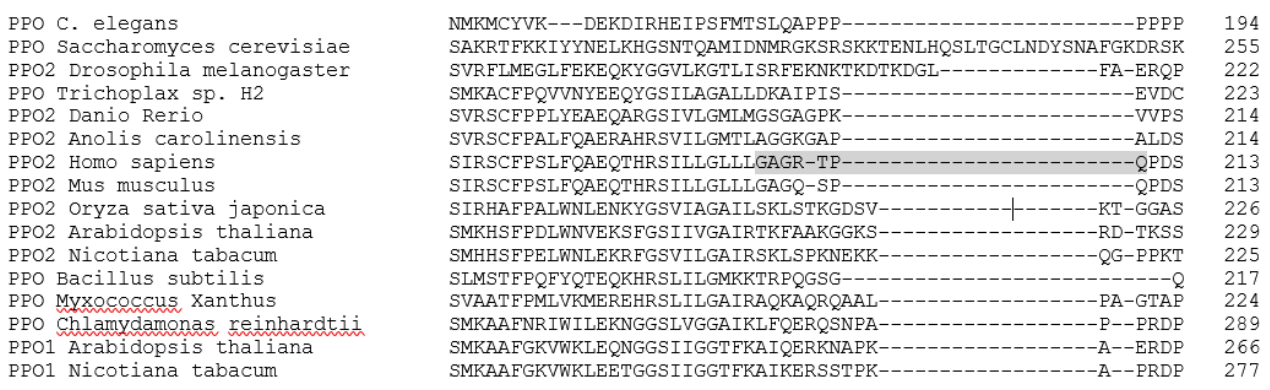

Figure 7. Alignment of 16 representative PPO sequences from model organisms with well characterized genomes, in the region of the unstructured loop. The loop from the human structure is highlighted. 


\section{Conclusions}

A novel docking model for protogen in the catalytic domain of PPO was created using a protogen structure derived from the crystal structure of a close precursor in the pathway, copro, bound to the catalytic domain of uroporphyrinogen decarboxylase. The intermediates in the pathway were created on the same backbone structure for docking, and the product proto was created based on previous crystallographic data. Binding energy for protogen was the most favorable with an average of $-10.59 \pm 1.08 \mathrm{kcal} / \mathrm{mol}$, and the intermediates and product showed binding energies $\sim 40 \%$ higher. The binding energies are consistent with the flexibility of the molecules, protogen being the most flexible and each intermediate thereafter reducing flexibility with the addition of double bonds. The docked molecules with the most energetically favorable positions all maintained an association between the propionate group of ring D and Arg98, which held the $\alpha-\mathrm{H}$ of $\mathrm{C} 10$ within the appropriate range for redox reactions to occur with the N5 of FAD (3.6-3.8 $)$ ). The findings here unify early experiments noting the hydride abstractions with the current understanding of the catalytic domain of PPO and known precursor conformations in the pathway. Finally, the unstructured loop near the opening to the catalytic domain was investigated for possible interaction with the substrate. A complete lack of homology, even within a phyla, indicates that no particular residue interacts with the substrate, but the conservation of the flexible region may indicate the necessity of accommodating substrate and product movement near the catalytic domain.

\section{Materials and Methods}

\subsection{Determining the Initial Conformation of Protogen and Construction of All the Reaction Intermediates}

Conformation of protogen was from derived from the structure of coproporphyrinogen III (copro) cocrystallized with uroporphyrinogen decarboxylase (1R3Y) [8]. This structure was extracted from the pdb file and saved as a mol2 file. As is common for ligand structures determined by protein crystallography, the atom types and bond orders of copro had to be reconstructed in silico using a molecular modelling and computational chemistry application (Spartan18, Wavefunction, Inc. Irvine, CA 92612). The bond angles and length were corrected by submitting the individual pyrrole rings to geometric minimization using density function theory calculations (wB97X-D 6-31*) (Supplemental Figure S7). The propionic acid groups found on rings A and B of the tetrapyrrole were converted to vinyl groups to convert copro into protogen. Once protogen was constructed, all the reaction intermediates $(1 \mathrm{a}, 1 \mathrm{c}, 2 \mathrm{a}, 2 \mathrm{~d})$ were built from this original confirmation by editing the mol2 files and submitted to geometric optimization with Spartan18. The structure of proto was obtained from the X-ray analysis of protoporphyrin IX dimethyl ester [14] deposited in The Cambridge Crystallographic Data Centre (CCDC) [28]. Spartan18 was used to remove the methyl esters and convert the propionic acids to propionate.

\subsection{Docking of Protogen, Tautomeric Reaction Intermediates and Proto to PPO}

The crystal structure of human PPO was obtained from 3nks [29]. The atom types of FAD were corrected using Spartan 18 and the ligand was converted to its oxidized form. The electrostatic charges were calculated using density function theory calculations (wB97X-D 6-31*).

Protogen and all of the reaction intermediates were docked into the catalytic domain of PPO using a modified Autodock method using Lennard-Jones potential and dummy atoms to overcome the software's limitation (AutoDock version 4.2, Scripps Institute, San Diego CA, USA) in handling flexible ring systems (Figure 3) $[12,13,30,31]$. This was done by editing the ligand.pdbqt files. Additionally, the guanidino group of arginine 97 was designated as important in the interaction between one of the propionate groups and a grid box was used to delimitate the region of the catalytic domain according to the software [13]. The gridbox dimensions were set to $90 \times 75 \times 75$ points with a spacing to 0.2 . The box was centered on the following coordinates: $x=-26.88, y=4.47$ and $z=45.17$. PPO was set as a rigid structure (Supplementary Figure S1). The cyclic tetrapyrroles modified to allow for flexible 
docking designed in the previous section were used for docking within the space defined in the gridbox. The algorithm was set to generate 50 docking poses and the top clustered was selected as optimal conformation for the docking of each ligand.

\subsection{Modeling of Disordered Loop Crossing the Opening of PPO Binding Domain}

The human PPO sequence was blasted in the NCBI database and the top 80 related sequences were selected. A second data set of 16 high quality PPO sequences spanning multiple phyla were selected to determine base homology (Supplemental Table S1). The two datasets were aligned using Clustal Omega [32]. The loop sequences from the more diverse dataset were compared from the alignment and then run through two predictive structure programs: Phyre2 [33] and PredictProtein [34].

Supplementary Materials: Supplementary Materials can be found at http://www.mdpi.com/1422-0067/21/24/ 9495/s1. Supplemental Figure S1. Gridbox defining the binding domain of the tetrapyrroles in PPO, including FAD forming the 'roof' of the cavity, the top of $\alpha-8$ helix of PPO upon which the tetrapyrrole rings are centered at the 'bottom' of the cavity, and arginine 97 involved in stabilizing the rings on the right side of the cavity. Supplemental Figure S2. Docking poses of the top cluster of intermediate 1a. Top = from above the catalytic domain of PPO and bottom = from inside the catalytic domain of PPO. Supplemental Figure S3. Docking poses of the top cluster of intermediate $1 \mathrm{c}$. Top $=$ from above the catalytic domain of PPO and bottom $=$ from inside the catalytic domain of PPO. Supplemental Figure S4. Docking poses of the top cluster of intermediate 2a. Top = from above the catalytic domain of PPO and bottom $=$ from inside the catalytic domain of PPO. Supplemental Figure S5. Docking poses of the top cluster of intermediate $2 \mathrm{~d}$. Top $=$ from above the catalytic domain of PPO and bottom $=$ from inside the catalytic domain of PPO. Supplemental Figure S6. Docking poses of the top cluster of intermediate proto. Top = from above the catalytic domain of PPO and bottom = from inside the catalytic domain of PPO. Supplemental Figure S7. DFT calculations on protoporphyrinogen IX pyrroles 1 and 2 in equilibrium geometry with DFT wB97XD 6-31G*. Supplemental table S1. Percent identity matrix of the Clustal Omega alignment made with the file of 16 model organisms protoporphyrinogen oxidase proteins.

Author Contributions: Conceptualization, methodology, and original draft preparation, A.L.B., H.B., and F.E.D. All authors have read and agreed to the published version of the manuscript.

Funding: This research was supported in part by Valent/Sumitomo, grant number 2006, Cotton Inc., Agreement No. 17-619 and the USDA National Institute of Food and Agriculture, Hatch Project 1016591, COL00785.

Acknowledgments: In this section you can acknowledge any support given which is not covered by the author contribution or funding sections. This may include administrative and technical support, or donations in kind (e.g., materials used for experiments).

Conflicts of Interest: The authors declare no conflict of interest.

\section{Abbreviations}

copro Coproporphyrinogen III

PPO Protoporphyrinogen oxidase

proto Protoporphyrin IX

protogen Protoporphyrinogen IX

\section{References}

1. Battersby, A.R. Tetrapyrroles: The pigments of life. Nat. Prod. Rep. 2000, 17, 507-526. [CrossRef]

2. Thunell, S. Porphyrins, porphyrin metabolism and porphyrias. I. Update. Scand. J. Clin. Lab. Investig. 2000, 60, 509-540. [CrossRef] [PubMed]

3. Mochizuki, N.; Tanaka, R.; Grimm, B.; Masuda, T.; Moulin, M.; Smith, A.G.; Tanaka, A.; Terry, M.J. The cell biology of tetrapyrroles: A life and death struggle. Trends Plant Sci. 2010, 15, 488-498. [CrossRef] [PubMed]

4. Dayan, F.E.; Barker, A.; Tranel, P.J. Origins and structure of chloroplastic and mitochondrial plant protoporphyrinogen oxidases: Implications for the evolution of herbicide resistance. Pest Manag. Sci. 2018, 74, 2226-2234. [CrossRef] [PubMed]

5. Wang, B.; Rudnick, S.; Cengia, B.; Bonkovsky, H.L. Acute hepatic porphyrias: Review and recent progress. Hepatol. Commun. 2019, 3, 193-206. [CrossRef] 
6. Jones, C.; Jordan, P.M.; Akhtar, M. Mechanism and stereochemistry of the porphobilinogen deaminase and protoporphyrinogen IX oxidase reactions: Stereospecific manipulation of hydrogen atoms at the four methylene bridges during the biosynthesis of haem. J. Chem. Soc. Perkin Trans. 1984, 1, 2625-2633. [CrossRef]

7. Koch, M.; Breithaupt, C.; Kiefersauer, R.; Freigang, J.; Huber, R.; Messerschmidt, A. Crystal structure of protoporphyrinogen IX oxidase: A key enzyme in haem and chlorophyll biosynthesis. EMBO J. 2004, 23, 1720-1728. [CrossRef]

8. Phillips, J.D.; Whitby, F.G.; Kushner, J.P.; Hill, C.P. Structural basis for tetrapyrrole coordination by uroporphyrinogen decarboxylase. EMBO J. 2003, 22, 6225-6233. [CrossRef]

9. Hao, G.-F.; Tan, Y.; Yang, S.-G.; Wang, Z.-F.; Zhan, C.-G.; Xi, Z.; Yang, G.-F. Computational and experimental insights into the mechanism of substrate recognition and feedback inhibition of protoporphyrinogen oxidase. PLoS ONE 2013, 8, e69198. [CrossRef]

10. Medlock, A.E.; Shiferaw, M.T.; Marcero, J.R.; Vashisht, A.A.; Wohlschlegel, J.A.; Phillips, J.D.; Dailey, H.A. Identification of the mitochondrial heme metabolism complex. PLoS ONE 2015, 10, 1-20. [CrossRef]

11. Ferreira, G.C.; Andrew, T.L.; Karr, S.W.; Dailey, H.A. Organization of the terminal two enzymes of the heme biosynthetic pathway. Orientation of protoporphyrinogen oxidase and evidence for a membrane complex. J. Biol. Chem. 1988, 263, 3835-3839. [PubMed]

12. Forli, S.; Botta, M. Lennard-Jones potential and dummy atom settings to overcome the AUTODOCK limitation in treating flexible ring systems. J. Chem. Inf. Model. 2007, 47, 1481-1492. [CrossRef] [PubMed]

13. Morris, G.M.; Huey, R.; Lindstrom, W.; Sanner, M.F.; Belew, R.K.; Goodsell, D.S.; Olson, A.J. AutoDock4 and AutoDockTools4: Automated docking with selective receptor flexibility. J. Comput. Chem. 2009, 30, 2785-2791. [CrossRef] [PubMed]

14. Caughey, W.S.; Ibers, J.A. Crystal and molecular structure of the free base porphyrin, protoporphyrin IX dimethyl ester. J. Am. Chem. Soc. 1977, 99, 6639-6645. [CrossRef]

15. Ismail, A.; Leroux, V.; Smadja, M.; Gonzalez, L.; Lombard, M.; Pierrel, F.; Mellot-Draznieks, C.; Fontecave, M. Coenzyme $\mathrm{Q}$ biosynthesis: Evidence for a substrate access channel in the FAD-dependent monooxygenase Coq6. PLoS Comput. Biol. 2016, 12, e1004690. [CrossRef]

16. Lennon, B.W.; Williams, C.H., Jr.; Ludwig, M.L. Crystal structure of reduced thioredoxin reductase from Escherichia coli: Structural flexibility in the isoalloxazine ring of the flavin adenine dinucleotide cofactor. Protein Sci. 1999, 8, 2366-2379. [CrossRef]

17. Eswaramoorthy, S.; Bonanno, J.B.; Burley, S.K.; Swaminathan, S. Mechanism of action of a flavin-containing monooxygenase. Proc. Natl. Acad. Sci. USA 2006, 103, 9832-9837. [CrossRef]

18. Stirling, A.J.; Gilbert, S.E.; Conner, M.; Mallette, E.; Kimber, M.S.; Seah, S.Y.K. A key glycine in bacterial steroid-degrading acyl-CoA dehydrogenases allows flavin-ring repositioning and modulates substrate side chain specificity. Biochemistry 2020, 59, 4081-4092. [CrossRef]

19. Lans, I.; Medina, M.; Rosta, E.; Hummer, G.; Garcia-Viloca, M.; Lluch, J.M.; González-Lafont, À. Theoretical study of the mechanism of the hydride transfer between ferredoxin-NADP ${ }^{+}$reductase and $\mathrm{NADP}^{+}$: The role of Tyr303. J. Am. Chem. Soc. 2012, 134, 20544-20553. [CrossRef]

20. Lans, I.; Peregrina, J.R.; Medina, M.; Garcia-Viloca, M.; González-Lafont, À.; Lluch, J.M. Mechanism of the hydride transfer between Anabaena Tyr303Ser FNRrd/FNRox and NADP+/H. A combined pre-steady-state kinetic/ensemble-averaged transition-state theory with multidimensional tunneling study. J. Phys. Chem. B 2010, 114, 3368-3379. [CrossRef]

21. Hammes-Schiffer, S. Comparison of hydride, hydrogen atom, and proton-coupled electron transfer reactions. ChemPhysChem 2002, 3, 33-42. [CrossRef]

22. Peters, K.S. A theory-experiment conundrum for proton transfer. Acc. Chem. Res. 2009, 42, 89-96. [CrossRef] [PubMed]

23. Walsh, C.T.; Wencewicz, T.A. Flavoenzymes: Versatile catalysts in biosynthetic pathways. Nat. Prod. Rep. 2013, 30, 175-200. [CrossRef] [PubMed]

24. Archipowa, N.; Kutta, R.J.; Heyes, D.J.; Scrutton, N.S. Stepwise hydride transfer in a biological system: Insights into the reaction mechanism of the light-dependent protochlorophyllide oxidoreductase. Angew. Chem. 2018, 130, 2712-2716. [CrossRef]

25. Piano, V.; Palfey, B.A.; Mattevi, A. Flavins as covalent catalysts: New mechanisms emerge. Trends Biochem. Sci. 2017, 42, 457-469. [CrossRef] 
26. Ghisla, S.; Massey, V. Mechanisms of flavoprotein-catalyzed reactions. In EJB Reviews 1989; Springer: Berlin/Heidelberg, Germany, 1989; pp. 29-45.

27. Valdar, W.S. Scoring residue conservation. Proteins 2002, 48, 227-241. [CrossRef]

28. Groom, C.R.; Bruno, I.J.; Lightfoot, M.P.; Ward, S.C. The Cambridge structural database. Acta Crystallogr. Sect. B 2016, 72, 171-179. [CrossRef]

29. Qin, X.; Tan, Y.; Wang, L.; Wang, Z.; Wang, B.; Wen, X.; Yang, G.; Xi, Z.; Shen, Y. Structural insight into human variegate porphyria disease. FASEB J. 2011, 25, 653-664. [CrossRef]

30. Forli, S.; Huey, R.; Pique, M.E.; Sanner, M.; Goodsell, D.S.; Olson, A.J. Computational protein-ligand docking and virtual drug screening with the AutoDock suite. Nat. Protoc. 2016, 11, 905-919. [CrossRef]

31. Lohning, A.E.; Levonis, S.M.; Williams-Noonan, B.; Schweiker, S.S. A practical guide to molecular docking and homology modelling for medicinal chemists. Curr. Top. Med. Chem. 2017, 17, 1-18. [CrossRef]

32. Madeira, F.; Park, Y.M.; Lee, J.; Buso, N.; Gur, T.; Madhusoodanan, N.; Basutkar, P.; Tivey, A.R.N.; Potter, S.C.; Finn, R.D.; et al. The EMBL-EBI search and sequence analysis tools APIs in 2019. Nucleic Acids Res 2019, 47, W636-W641. [CrossRef] [PubMed]

33. Kelley, L.A.; Mezulis, S.; Yates, C.M.; Wass, M.N.; Sternberg, M.J.E. The Phyre2 web portal for protein modeling, prediction and analysis. Nat. Protoc. 2015, 10, 845-858. [CrossRef] [PubMed]

34. Yachdav, G.; Kloppmann, E.; Kajan, L.; Hecht, M.; Goldberg, T.; Hamp, T.; Hönigschmid, P.; Schafferhans, A.; Roos, M.; Bernhofer, M. PredictProtein-an open resource for online prediction of protein structural and functional features. Nucleic Acids Res 2014, 42, W337-W343. [CrossRef] [PubMed]

Publisher's Note: MDPI stays neutral with regard to jurisdictional claims in published maps and institutional affiliations. 